\title{
CONTACT PROBLEM FOR THE RIGID RING STAMP AND THE HALF-SPACE WITH INITIAL (RESIDUAL) STRESSES
}

\author{
N. F. Yaretskaya
}

\begin{abstract}
The problem of the contact interaction of a rigid cylindrical ring punch and half-space with initial (residual) stresses is considered disregarding the friction forces in the case of unequal roots of the characteristic equation. The study is performed in common form for the theory of large (finite) initial deformations and two variants of the theory of small initial deformations within the framework of the linearized theory of elasticity for arbitrary elastic potential. The numerical results are presented in the form of graphs for Treloar's potential.
\end{abstract}

Keywords: linearized theory of elasticity, initial (residual) stresses, contact problem, ring punch, half-space

Introduction. Applied problems of modern engineering and new technologies associated with the necessity of predicting the contact behavior of different structures stimulated recently the development of various mathematical models and methods of contact mechanics for bodies with different properties $[9,12,13]$.

The effect of initial (residual) stresses, which are always present is real structures and machine elements, is one of the important factors of contact interaction. Therefore, the development of efficient methods of computation of the stress-strain state by considering initial deformations $[1,2,4-7,14,15]$ is an urgent and significant scientific and engineering problem. There are also a number of other generalizing publications $[10,11,16]$ fully or partially related to the subject of the present article.

A fairly detailed review of works related to the contact pressure of rigid punches (including ring ones) in the absence of initial stresses is presented in the monograph [8].

Contact interaction of rigid and elastic punches with prestressed bodies was studied in [1, 2, 4-7, 13, 15]. Here either elastic potentials of definite structure were considered or the problem was stated in general form for compressible (incompressible) bodies with arbitrary potential based on the linearized theory of elasticity. The contact problem for a rigid ring punch of complex configuration forced into an elastic layer with initial stresses was solved in [2] using harmonic potential. The punch was formed by rotating about a common axis two branches of parabolas connected at the vertices by a straight line perpendicular to the axis of rotation (the axes of the parabolas bounding the punch are parallel to the common axis of rotation).

In the present article, the axisymmetric spatial contact problem for a rigid ring punch with plane base and a half-space with initial (residual) stresses without friction (for unequal roots of the governing equation [7]) is solved using the linearized theory of elasticity $[4-7,13,15]$. The study is performed in common form for compressible and incompressible bodies for the theory of large initial deformations and two variants of the theory of small initial deformations for arbitrary elastic potential.

Assume that the initial stress-strain state in the half-space is homogeneous.

1. Problem Statement. Basic Equations. Let a finite rigid ring punch with plane base whose geometrical axis of symmetry coincides with the axis $y_{3}$ of the cylindrical coordinate system $\left(r, \theta, y_{3}\right)$ be forced into the half-space (Fig. 1$)$ by a force $P$ (after occurrence of the initial strain state); $R_{1}, R_{2}$ are the inner and outer radii of the punch. Let the external loading be applied only to the free edge of the punch so that all its points displace along the axis of symmetry $y_{3}$ by the same value $\varepsilon$. Let also the

Khmelnitskiy National University, 11 Institutskaya St., 29016 Khmelnitskiy, Ukraine; e-mail: massacran2@ukr.net. Translated from Prikladnaya Mekhanika, Vol. 54, No. 5, pp. 55-60, September-October, 2018. Original article submitted July 2, 2017. 


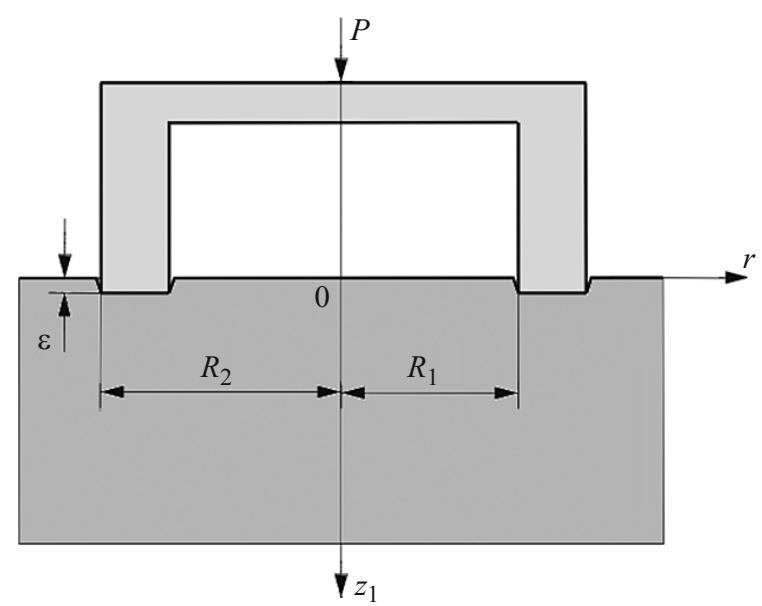

Fig. 1

surfaces outside the contact zone remain to be free from the influence of the external forces and the displacements and stresses in the contact zone be continuous. Note also that a similar problem for a layer was addressed in [2].

Assume that the initial state of the half-space is homogeneous and the following relations hold [7, 15]:

$$
y_{m}=x_{m}+U_{m}^{0}, \quad U_{m}^{0}=\delta_{m i}\left(\lambda_{m}-1\right) \lambda_{i}^{-1} y_{i} \quad(i, m=\overline{1,3}) .
$$

Then, the main equation for displacements $[7,15]$ for compressible bodies has the form

$$
L_{m \alpha}^{\prime} U_{\alpha}=0, \quad L_{m \alpha}^{\prime}=\omega_{i j \alpha \beta}^{\prime} \partial^{2} / \partial y_{i} y_{\beta} \quad(i, m, \alpha, \beta=\overline{1,3}),
$$

The incompressibility condition is satisfied for incompressible bodies:

$$
\begin{gathered}
L_{m \alpha}^{\prime}=\kappa_{i m \alpha \beta}^{\prime} \partial^{2} / \partial y_{i} y_{\beta}, \quad L_{m \alpha}^{\prime} U_{\alpha}+q_{\alpha m}^{\prime} \partial p^{\prime} / \partial y_{\alpha}=0, \\
q_{i j}^{\prime} \partial U_{j} / \partial y_{i}=0, \quad q_{i j}^{\prime}=\lambda_{i} q_{i j} \quad(i, j, m, \alpha, \beta=\overline{1,3}) .
\end{gathered}
$$

The expressions for the components of the stress tensor for compressible and incompressible bodies are

$$
\begin{gathered}
Q_{i j}^{\prime}=\omega_{i j \alpha \beta}^{\prime} \frac{\partial U_{\alpha}}{\partial y_{\beta}}, \quad Q_{i j}^{\prime}=\kappa_{i j \alpha \beta} \frac{\partial U_{\alpha}}{\partial y_{\beta}}+q_{i j}^{\prime} p, \\
\omega_{i j \alpha \beta}^{\prime}=\frac{\lambda_{i} \lambda_{\beta}}{\lambda_{1} \lambda_{2} \lambda_{3}} \omega_{i j \alpha \beta}, \quad \kappa_{i j \alpha \beta}^{\prime}=\frac{\lambda_{i} \lambda_{\beta}}{\lambda_{1} \lambda_{2} \lambda_{3}} \kappa_{i j \alpha \beta} .
\end{gathered}
$$

For homogeneous initial stresses, we assume that the conditions $S_{0}^{11}=S_{0}^{22} \neq 0, S_{0}^{33}=0, \lambda_{1}=\lambda_{2} \neq \lambda_{3}$ are met. Then the solutions of Eqs. (1.1), (1.2) in the cylindrical coordinates $\left(r, \theta, y_{3}\right)$ are represented in the form

$$
\left(\Delta_{1}+\xi_{2}^{\prime 2} \partial^{2} / \partial y_{3}^{2}\right)\left(\Delta_{1}+\xi_{3}^{\prime 2} \partial^{2} / \partial y_{3}^{2}\right) \tilde{\chi}=0,
$$

where $\Delta_{1}=\partial^{2} / \partial r^{2}+r^{-1} \partial / \partial r$.

As mentioned above, we restrict ourselves to the case of unequal roots $\left(\xi_{2}^{\prime 2} \neq \xi_{3}^{\prime 2}\right)$ of Eq. (1.3).

In the system of circular cylindrical coordinates $\left(r, \theta, z_{i}\right)$, where $z_{i}=v_{i}^{-1} y_{3}, v_{i}=\sqrt{n_{i}}(i=1,2), n_{1}=\xi_{2}^{\prime 2}, n_{2}=\xi_{3}^{\prime 2}$, the boundary conditions are the following: 